\title{
The Role of Microglia in Inherited White-Matter Disorders and Connections to Frontotemporal Dementia
}

This article was published in the following Dove Press journal: The Application of Clinical Genetics

\begin{abstract}
Microglia play a critical but poorly understood role in promoting white-matter homeostasis. In this review, we leverage advances in human genetics and mouse models of leukodystrophies to delineate our current knowledge and identify outstanding questions regarding the impact of microglia on central nervous system white matter. We first focus on the role of pathogenic mutations in genes, such as TREM2, TYROBP, and CSF1R, that cause leukodystrophies in which the primary deficit is thought to originate in microglia. We next discuss recent advances in disorders such as adrenoleukodystrophy and Krabbe disease, in which microglia play an increasingly recognized role. We conclude by reviewing the roles of $G R N$ and related genes, such as TMEM106B, PSAP, and SORT1, that affect microglial biology and associate with several types of disease, including multiple leukodystrophies as well as forms of frontotemporal dementia (FTD) presenting with white-matter abnormalities. Taken together, mouse and human data support the notion that loss of microglia-facilitated white-matter homeostasis plays an important role in the development of leukodystrophies and suggest novel mechanisms contributing to FTD.
\end{abstract}

Keywords: leukodystrophies, leukoencephalopathies, frontotemporal dementia, white matter, microglia, progranulin

\section{Introduction}

Leukodystrophies include a vast group of rare, multifarious genetic disorders that selectively and primarily affect the central nervous system (CNS) white matter. These disorders encompass defects in the generation, maintenance, and repair of white matter, and the primary molecular deficit may arise not only in myelinproducing oligodendrocytes but also in astrocytes, microglia, or other cell types. $^{1-3}$ Several informative reviews published in the last several years provide broad overviews of inherited diseases of white matter, including those focused on childhood $^{4}$ and adult-onset ${ }^{5,6}$ disorders. Here we will focus primarily on leukodystrophies that are apparently caused by primary microglial defects, disorders that are sometimes termed microgliopathies. ${ }^{7}$

Microglia are macrophages of the brain parenchyma that are now understood to play essential roles in brain development, homeostasis, inflammation, and neurodegeneration. ${ }^{8,9}$ The particular importance of microglia in promoting the health and resilience of CNS white matter has emerged in the 21 st century due in large part to the identification of pathogenic mutations in microglia-expressed genes in Mendelian white-matter disorders.
Correspondence: Jennifer S Yokoyama Memory and Aging Center, Department of Neurology, University of California San Francisco, 675 Nelson Rising Lane, Suite 190, San Francisco, CA, 94158, USA

Tel +I 4 I5 476-5565

Fax +I 415 502-7588

Email jennifer.yokoyama@ucsf.edu
The Application of Clinical Genetics 202 I:|4 195-207 in - 
In the first section, we leverage work in human and mouse genetics to describe the primary microgliaassociated leukodystrophies, which are caused by pathogenic mutations in genes such as TREM2 (encoding the triggering receptor expressed on myeloid cells 2), TYROBP (TYRO protein tyrosine kinase-binding protein), CSF1R (colony-stimulating factor 1 receptor), and USP18 (ubiquitin-specific protease 18). Building on these findings, we transition our focus toward diseases in which microglia play an increasingly recognized role and explore recent advances in our understanding of white-matter microglia. Our overarching goal in exploring these disorders and their genetic causes is to synthesize a more robust understanding of the mechanisms by which microglia maintain CNS white-matter homeostasis, not only after acute whitematter insult but also over the entire lifespan and in disease. Finally, we highlight a new frontier in the study of leukodystrophies: a small group of genes associated with the expression and/or function of the secreted glycoprotein, progranulin. Members of this group of genes influence lysosomal function, ${ }^{10,11}$ shape microglial biology in important ways, ${ }^{12-14}$ and are causally involved in several distinct forms of leukodystrophy ${ }^{15,16}$ as well as early-onset neurodegenerative disease resulting in a clinical syndrome of frontotemporal dementia (FTD) ${ }^{17,18}$ Intriguingly, the FTD cases associated with this group of genes (including $G R N$ [encoding progranulin], TMEM106B [transmembrane protein 106B] and SORT1 [sortilin]) show evidence of whitematter changes that are otherwise atypical for FTD. ${ }^{19-22}$

\section{Primary Microglial Leukodystrophies Nasu-Hakola Disease: Role of TYROBP and TREM2}

Nasu-Hakola disease, also known as polycystic lipomembranous osteodysplasia with sclerosing leukoencephalopathy (PLOSL), was first associated with pathogenic mutations in TYROBP (encoding a protein often called DAP12 [DNAXactivating protein of $12 \mathrm{kDa}$ ]) approximately 20 years ago. ${ }^{23}$ Shortly after this discovery, additional Nasu-Hakola patients harboring pathogenic mutations in TREM2 were identified. ${ }^{24}$ Given that TREM2 is a microglial receptor that interacts with and signals via DAP $12,{ }^{25}$ the identification of loss-offunction mutations in the genes encoding both of these proteins - which cause the same recessively inherited disorder provided some of the first strong evidence that aberrant microglia function could cause an adult-onset leukodystrophy.
Nasu-Hakola disease classically involves phenotypes beyond loss of myelin and cerebral axons, including formation of bone cysts and basal ganglia calcification; ${ }^{26,27}$ however, several cases of early-onset FTD-like syndromes involving white-matter loss but lacking overt bone phenotypes have also been associated with loss-of-function mutations in TREM2. ${ }^{28-30}$ Consideration of these cases suggests that while loss of TREM2 function in osteoclasts can in some cases be compensated for, TREM2 function in microglia appears to be essential for the maintenance of white matter throughout the lifespan. In addition to these early-onset FTD-like syndromes associated with complete or near-complete loss of TREM2 function, rare heterozygous variants in TREM2 are also thought to increase risk for $\mathrm{FTD}^{31,32}$ (in addition to their well-established role in increasing risk for Alzheimer disease ${ }^{33,34}$ ), although it remains unclear if partial loss of TREM2 function increases risk for FTD via loss of white-matter integrity, reduced microglial clearance of pathological proteins more typically associated with FTD (eg, tau and TDP-43), or a combination of these or other mechanisms.

TREM2 binds a variety of lipidic ligands including anionic and zwitterionic phospholipids, bacterial lipopolysaccharide, and myelin-enriched lipids, such as sulfatide and sphingomyelin (reviewed in $^{25,35-37}$ ). In addition, TREM2 can interact with several protein ligands including apolipoproteins (eg, APOE) and amyloid- $\beta .^{25}$ Given TREM2's ability to sense myelin-derived lipids, and the known role of TREM2, TYROBP, and microglia in leukodystrophies, it is reasonable to hypothesize that proper microglial maintenance of white-matter homeostasis involves direct sensing of myelin-derived components and subsequent signal transduction via a functional TREM2-DAP12 complex. Indeed, several papers employing cuprizone-induced demyelination in mice lacking Trem2 support this possibility. ${ }^{38,39}$ More recent work suggests that loss of Trem2 specifically leads to pathological cholesteryl ester accumulation in microglia downstream of myelin debris phagocytosis in a chronic demyelination model. ${ }^{40}$ Encouragingly, activation of Trem2 in vivo with an agonistic antibody enhances myelin debris clearance after cuprizone treatment and promotes the repopulation of oligodendrocytes, subsequent remyelination, and partial protection against axonal damage. ${ }^{41}$ Collectively, mouse models of Trem 2 deficiency suggest a role for microglial Trem2/Dap12 in maintaining white-matter health by (i) sensing myelin-derived lipids that result from myelin damage; (ii) generating signaling cascades 
that promote phagocytosis of debris; (iii) enabling homeostatic metabolism and clearance of myelin-derived cholesterol; and (iv) promoting recruitment of the oligodendrocyte precursor cells (OPCs) that are required for remyelination and, ultimately, preservation of axonal integrity.

\section{ALSP: Role of CSFIR and AARS2}

Formerly considered to be two distinct clinical entities, hereditary diffuse leukoencephalopathy with axonal spheroids (HDLS) and pigmented orthochromatic leukodystrophy (POLD) have been unified into a single clinicopathologic entity - adult-onset leukoencephalopathy with axonal spheroids and pigmented glia (ALSP) - with the identification of CSF1R mutations in both disorders. ${ }^{42-45}$ ALSP is a progressive and clinically heterogeneous disorder with antemortem diagnoses from early family studies including FTD, Alzheimer disease, and even multiple sclerosis. ${ }^{43}$ Histologically, the disorder is characterized by degeneration of white matter and axons as well as the presence of pigment-laden macrophages. ${ }^{45}$ Given that CSF1R is expressed on microglia within the brain and CSF1R signaling is essential for the development of microglia, ${ }^{46,47}$ ALSP due to CSF1R mutations can be considered to be a primary microglial leukodystrophy. ${ }^{1,48}$ ALSP caused by CSF1R mutations is inherited in an autosomal-dominant manner, with many of the described mutations abrogating autophosphorylation within the intracellular tyrosine kinase domain. ${ }^{43,44}$ The presence of heterozygous loss-of-function mutations in ALSP suggests that haploinsufficiency of CSF1R signaling is sufficient to cause severe adult-onset white-matter degeneration downstream of microglial dysfunction. ${ }^{48}$ Interestingly, recent work from our institution highlights hematopoietic stem cell transplantation (HSCT) as a potentially promising clinical therapy for ALSP, with both patients in the study demonstrating partial clinical stabilization and reduced white-matter abnormalities on brain MRI. ${ }^{49}$ The findings from this case study are consistent with the possibility that transplant-derived myeloid cells are capable populating the microglial niche and restoring CSF1R signaling.

The recent identification of homozygous CSF1R mutations in childhood-onset leukodystrophy involving agenesis of the corpus callosum ${ }^{50}$ not only underscores the importance of microglial CSF1R signaling in white-matter maintenance but further suggests a role for microglia in supporting the development of CNS white matter. Work from mouse models suggests that interleukin 34 (IL-34), rather than CSF-1, is the critical CSF1R ligand enabling the downstream signaling that is necessary for microglial development and/or maintenance. ${ }^{47,51}$ Recent work in zebrafish has suggested that brain-derived IL-34 drives the recruitment of embryonic macrophages (ie, microglia precursors) into the $\mathrm{CNS},{ }^{52}$ although prior work in mice has suggested that IL-34 may be particularly important for maintenance (rather than development) of microglia in the mammalian brain. ${ }^{51}$ Considering our current knowledge of IL-34-CSF1R signaling, the future discovery of $l L 34$ mutations in otherwise unexplained cases of leukodystrophy would not be unexpected.

After the discovery of CSF1R mutations as a cause of ALSP, additional cases remained that lacked such mutations. Some of these individuals were subsequently found to harbor compound heterozygous or homozygous loss-offunction mutations in $A A R S 2$, encoding mitochondrial alanyl-tRNA synthetase $2 .^{53-55}$ It is noteworthy that mutations in $A A R S 2$, encoding a protein with a function unrelated to that of CSF1R - whose expression is ubiquitous rather than restricted to the myeloid lineage - can result in an adultonset leukodystrophy resembling ALSP (albeit with additional phenotypes, such as ovarian failure in women). Additional research is needed to determine how loss of a seemingly disparate biochemical function can promote such a clinically similar phenotype.

\section{Pseudo-TORCH Syndrome and the Role of USPI8 in Microglia}

Pseudo-TORCH (toxoplasmosis, other infections, rubella, cytomegalovirus, and herpes simplex virus) syndrome due to loss-of-function mutations in USP 18 represents an interferonopathy and microgliopathy resulting in white-matter damage in mice and humans. ${ }^{56-58}$ Elucidation of the role of USP18 - a multifunctional protein that possesses both isopeptidase activity and inhibitory activity toward type I interferon (IFN) signaling ${ }^{59}$ - in microglia suggests that loss of a crucial inflammation-dampening mechanism is sufficient to produce CNS pathology. In particular, the derepression of signaling resulting in the expression of IFNstimulated genes in microglia appears likely to be a culprit in the microglia-mediated destruction of white matter. ${ }^{60}$

Integrating the literature on Nasu-Hakola disease and ALSP with USP18-mediated pseudo-TORCH syndrome suggests that microglia can cause destructive whitematter disease in at least two apparently opposing manners: (i) loss of beneficial signaling required to promote 
microglial survival, proliferation, and metabolism prevents microglia from appropriately responding to and clearing incipient white-matter damage (eg, via loss of TREM2DAP12 or CSF1R-DAP12 signaling); and (ii) loss of negative regulation of the type I IFN pathway unleashes toxic inflammatory processes leading to white-matter pathology (eg, via loss of USP18 negative regulation). Additional mechanisms are likely to be uncovered in the coming years. Despite the fact that Usp 18 expression is enriched in white-matter microglia, ${ }^{56}$ it is not fully clear why USP18-associated disease appears to selectively affect microglia in the white matter; future studies will be needed to address this issue.

\section{Microglia-Modulated}

\section{Leukodystrophies and} White-Matter Microglia

\section{Microglia as Previously Unappreciated Modulators of Leukodystrophies}

Microglia as Drivers of Neuronal Death and Disease Progression in Adrenoleukodystrophy

Adrenoleukodystrophy occurs due to peroxisomal dysfunction that causes very long chain fatty acid (VLCFA) accumulation in all tissues due to an X-linked mutation in $A B C D 1$ (encoding ATP-binding cassette D1, which transports VLCFA into peroxisomes). ${ }^{61}$ The clinical phenotype is highly variable, but usually includes early-onset adrenal insufficiency (median time to glucocorticoid replacement therapy $\sim 16$ years) and a variable neurological phenotype with cerebral and/or spinal cord demyelination (median time to cerebral disease $\sim 35$ years). ${ }^{62}$ The neurological phenotype increases in prevalence as a patient ages, with $\sim 80 \%$ of patients surviving past age 64 having cerebral disease. Historical work has focused largely on VLCFA toxicity in oligodendroglia given their high lipid content, ${ }^{61}$ but the central role of microglia in cerebral and spinal demyelination is becoming increasingly apparent. For example, recent work in mouse models of adrenoleukodystrophy has found that microglial activation preceded synaptic loss and that $A b c d 1$-deficient microglia demonstrated a pro-phagocytotic phenotype with upregulated Trem2 expression. ${ }^{63}$ Analysis of brain tissue samples from adrenoleukodystrophy patients by Bergner et al support this finding - prelesional areas were remarkable for only minimal changes to oligodendroglial and neuronal morphology but with microglia showing signs of activation, including decreased TMEM119 expression and conversion to an amoeboid phenotype. ${ }^{64}$ This and prior studies also demonstrate microglial depletion in prelesional areas compared to healthy white matter and demyelinated regions, suggesting that activated microglia may undergo apoptosis just prior to demyelination. ${ }^{64,65}$ Of note, a similar pattern of microglial depletion prior to demyelination has also been observed in metachromatic leukodystrophy, a disorder caused by mutations in ARSA encoding the lysosomal enzyme arylsulfatase A that breaks down sulfatides - and, less frequently, in PSAP which encodes prosaposin and will be discussed in detail below in the context of progranulin function. ${ }^{16,64}$

Treatment of adrenoleukodystrophy is distinctive amongst leukodystrophies and further highlights the importance of immune cells in the pathophysiology of neurodegeneration secondary to $A B C D 1$ mutations. If given early, bone marrow transplantation has been shown to slow or halt the progression of adrenoleukodystrophy. ${ }^{66}$ More recently, the therapy has been refined further and autologous transplants of $\mathrm{CD} 34+$ cells with a functioning copy of $A B C D 1$ now show promise in clinical trials ${ }^{67}$ with similar results to a conventional bone marrow transplant but notably without signs of graft-versus-host disease or other transplant-related complications. This development is of clinical and scientific significance because it suggests that the disease-modifying aspects of CD34+ transplants may be due to the impact of properly functioning macrophages (which can migrate into the brain and potentially fill the microglial niche) rather than the immunosuppressive medications required after conventional HSCT.

\section{Microglia in Krabbe Disease: Drivers of} Demyelinating Disease or Innocent Bystanders?

Krabbe disease, also known as globoid cell leukodystrophy, occurs in patients with deficiency of the lysosomal enzyme galactocerebrosidase (encoded by GALC). ${ }^{68,69}$ Patients with Krabbe disease accumulate both galactosylceramide and galactosylsphingosine (also known as psychosine), leading to widespread demyelination with reactive gliosis remarkable for both multinucleated microglia (globoid cells) and astrocytosis. ${ }^{70,71}$ Interestingly, in vitro work has shown that psychosine alone is sufficient to produce a globoid cell-like phenotype ${ }^{72}$ suggesting that globoid-cell formation may occur independently of oligodendroglial death rather than as a reaction to it. Adding to the intrigue of this finding is evidence from new mouse models of Krabbe disease that demonstrate early gliosis, globoid cell formation, and elevated psychosine levels 
prior to cell death and in the absence of substantial demyelination. ${ }^{73}$ While psychosine is a known oligodendroglial toxin, ${ }^{74}$ the possibility that aberrant microglial function could contribute to white-matter disease is supported by data from fetal human tissue with elevated psychosine, confirming the presence globoid cells without concurrent demyelinating disease. ${ }^{75}$

The potential import of this finding is heightened by the fact that immunomodulation in the form of HSCT is the most effective disease-modifying therapy for Krabbe disease. ${ }^{76,77}$ As in adrenoleukodystrophy, HSCT is more effective when given prior to the onset of clinical symptoms and sometimes associated with reduced white-matter disease on T2-weighted imaging. ${ }^{77,78}$ Although the precise therapeutic mechanisms responsible for the relative success of HSCT in Krabbe disease remain unknown, these findings on balance suggest that the microglial contributions to Krabbe disease pathophysiology - including demyelination in particular - may be underappreciated and that future research will be required to determine the specific mechanisms by which microglia modulate and possibly even drive aspects of demyelinating pathology.

\section{Advances in Our Understanding of White-Matter Microglia}

How do microglia promote white-matter homeostasis in health and disease? A variety of novel mouse models have refined our knowledge of microglia residing in the white matter and suggest additional relevant mechanisms. For example, microglial transglutaminase-2 activity supports the survival of OPCs and promotes both developmental myelination and remyelination. ${ }^{79}$ This finding bolsters the notion that microglial support of white-matter physiology is not merely a function of sensing and clearing nascent whitematter damage, but rather involves active trophic support of oligodendroglial cells. Studies of remyelination using the optic nerve crush model indicate that microglial activation soon after injury is crucial for robust OPC proliferation but ultimately inhibits the differentiation of these precursor cells into mature, myelination-competent oligodendrocytes. ${ }^{80}$ Accordingly, depletion of microglia using a small-molecule CSF1R inhibitor several weeks after injury (but not earlier) enabled differentiation of recently generated OPCs, and in conjunction with inhibition of the oligodendrocyte $G$ proteincoupled receptor (GPR) 17, enabled remyelination of the injured optic nerve. ${ }^{80}$
In contrast to the supportive role that microglia can play in myelination, several recent papers indicate that disrupted transforming growth factor (TGF)- $\beta$ signaling in microglia as well as peripheral monocytes capable of colonizing the CNS as tissue-resident macrophages under defined conditions - can result in potent white-matter destruction. ${ }^{81,82}$ Among other effects, loss of microglial TGF- $\beta$ signaling results in impaired OPC differentiation into mature, myelinproducing oligodendrocytes. ${ }^{83}$ On balance, this body of literature highlights that, while microglia can - in specific contexts - provide essential support to oligodendroglial cells, they also possess a latent, tightly regulated potential to engage in highly pathogenic behavior in the white matter.

In addition to the above hypothesis-driven studies, large-scale, single-cell RNA sequencing studies have revealed a transient subpopulation of microglia localizing to early postnatal white matter ${ }^{84}$ and a population of microglia associated with aging white matter. ${ }^{85}$ Given that the early postnatal microglial subpopulation is observed in the developing corpus callosum and that children with homozygous, loss-of-function CSF1R mutations show agenesis of the corpus callosum (described above ${ }^{50}$ ), it is reasonable to speculate that specialized, developing white-matter microglia may be conserved in humans and involved in the proper development of CNS white matter.

\section{Progranulin-Associated Genes: Role in Disorders of White Matter \\ Progranulin}

Haploinsufficiency of the secreted glycoprotein progranulin, encoded by $G R N$, was first linked to familial frontotemporal lobar degeneration (FTLD) characterized by pathologic TAR DNA-binding protein (TDP)-43 inclusions in 2006. ${ }^{17,18}$ Other common causes of familial FTLD include pathogenic hexanucleotide repeat expansion intronic to C9orf72 (chromosome 9 open reading frame 72) and pathogenic mutations in MAPT (microtubule-associated protein tau). ${ }^{86}$ Of note, FTLD cases attributable to GRN, C9orf72, and MAPT demonstrate gene-specific white-matter changes measured using diffusion tensor imaging (DTI) ${ }^{87,88}$ However, pathogenic GRN mutations are further differentiated among these common causes of familial FTLD in that a subset of cases (13-20\%) demonstrate substantial white-matter hyperintensities (WMH) beyond the DTI and gray-matter changes seen across the spectrum of neurodegenerative phenotypes with TDP-43 and tau neuropathology (Table 1). ${ }^{19,20,89}$ This finding's relevance to leukodystrophies is further heightened by 
Table I Progranulin (GRN)-Related Genes Associated Directly or Indirectly with Frontotemporal Dementia (FTD) and Inherited White-Matter Disorders

\begin{tabular}{|l|l|l|l|l|l|l|}
\hline $\begin{array}{l}\text { Gene } \\
\text { Symbol }\end{array}$ & $\begin{array}{l}\text { Expressed } \\
\text { in } \\
\text { Microglia? }\end{array}$ & $\begin{array}{l}\text { Enriched } \\
\text { in } \\
\text { Microglia? }\end{array}$ & $\begin{array}{l}\text { Affects GRN } \\
\text { Trafficking or } \\
\text { Circulating Levels? }\end{array}$ & $\begin{array}{l}\text { Genetic } \\
\text { Association } \\
\text { with FTD? }\end{array}$ & $\begin{array}{l}\text { Genetic Association with } \\
\text { White-Matter Disease? }\end{array}$ & $\begin{array}{l}\text { Selected } \\
\text { References }\end{array}$ \\
\hline GRN & Yes & Yes & N/A & Causative & $\begin{array}{l}\text { Subset of FTLD-GRN shows } \\
\text { WMH }\end{array}$ & {$[17-20,89-91,93]$} \\
\hline TMEMI06B & Yes & No & Circulating levels & Risk modifier & $\begin{array}{l}\text { Hypomyelinating } \\
\text { leukodystrophy }\end{array}$ & {$[15,20,94-97,102-105]$} \\
\hline PSAP & Yes & Yes & $\begin{array}{l}\text { Trafficking and } \\
\text { circulating levels }\end{array}$ & Not yet & $\begin{array}{l}\text { Metachromatic } \\
\text { leukodystrophy; other } \\
\text { sphingolipidoses }\end{array}$ & {$[16,111,115,118]$} \\
\hline SORTI & Yes & No & $\begin{array}{l}\text { Trafficking and } \\
\text { circulating levels }\end{array}$ & Risk factor & $\begin{array}{l}\text { Subset of FTD cases harboring } \\
\text { rare SORTI variants present } \\
\text { with WMH }\end{array}$ & {$[22,119]$} \\
\hline
\end{tabular}

Notes: The GRN-associated genes TMEMIO6B, PSAP, and SORTI are associated with circulating progranulin levels and in some cases also influence progranulin sorting. Most of the above genes are genetically associated with FTD as well as various forms of leukodystrophy or white-matter hyperintensities in the context of frontotemporal lobar degeneration pathology. These findings, when considered collectively, suggest that variation in the PSAP locus may ultimately be identified as a risk factor for FTD. Moreover, the findings suggest that progranulin-associated proteins may impart risk for FTD by modulating microglial function and white-matter resilience over the lifespan.

Abbreviations: FTD, frontotemporal dementia; FTLD, frontotemporal lobar degeneration; GRN, progranulin; PSAP, prosaposin; SORTI, sortilin; TMEMI06B, transmembrane protein I06B; WMH, white-matter hyperintensities.

the absence of other potential explanations for the substantial WMH observed in FTLD-GRN patients, such as vascular disease or major vascular risk factors, mitochondrial or metabolic disease, or other neuroinflammatory conditions. ${ }^{19,90}$ A recent case report provided additional insight into this discovery, demonstrating that the WMH seen on MRI are associated with marked microgliosis but only mild axonal loss and minimal vascular disease. ${ }^{91}$ Taken together, these observations suggest that the white-matter findings reported for FTLD-GRN patients are likely specific to the microglial dysfunction caused by $G R N$ mutations rather than other, more established, causes of WMH.

At the subcellular level, progranulin appears to be particularly important for maintaining lysosomal homeostasis. ${ }^{10}$ Progranulin - which is synthesized as a precursor protein that can be proteolyzed into peptides termed granulins - is sorted to the lysosome by virtue of its interaction with sortilin and another secreted glycoprotein, prosaposin (see below), although it remains unclear precisely which aspects of lysosomal function progranulin regulates once delivered to the lysosome. ${ }^{10}$ One intriguing model suggests that partial or complete loss of progranulin results in reduced delivery of prosaposin to the neuronal lysosome, which in turn would lead to impaired glycosphingolipid metabolism. ${ }^{92}$

At the cellular level, loss of progranulin affects microglial biology in numerous ways. For example, loss of Grn in mice results in pathologic activation of microglia during aging in a process that involves inappropriate complement production. ${ }^{12,14}$ In addition, microglia-specific deletion of Grn results in specific behavioral alterations downstream of aberrant microglial activation of nuclear factor (NF)- $\mathrm{kB}$ and tumor necrosis factor (TNF)- $\alpha .{ }^{13}$ Interestingly, despite the fact that loss-of-function mutations in both $G R N$ and TREM2 are associated with neurodegeneration and whitematter pathology - and that both genes regulate microglial physiology - a direct comparison of the transcriptomes of microglia lacking either Grn or Trem 2 revealed highly divergent microglial transcriptional profiles. ${ }^{93}$ In particular, loss of Trem 2 results in increased expression of so-called homeostatic microglial genes and decreased expression of disease-associated genes, whereas the opposite profile is observed in Grn-null microglia. ${ }^{93}$

A careful consideration of the role of $G R N$ and TREM2 in shaping microglial biology therefore further supports the notion that neurodegeneration and whitematter damage downstream of pathogenic mutations in microglia-expressed genes are unlikely to result from a single, monolithic shift in microglial physiology. Rather, the data here once again suggest a more likely scenario in which both the inability of microglia to respond appropriately to incipient cellular damage as well as chronic hyperactivation of microglia can similarly result in downstream white-matter damage and neuropathology. 


\section{TMEMI06B}

TMEM106B, encoding a transmembrane protein that localizes primarily to lysosomes, represents an important genetic modifier of FTLD risk due to pathogenic GRN mutations, ${ }^{94-96}$ and the protective allele of TMEM106B is associated with increased plasma levels of progranulin. ${ }^{94,95}$ Carriers of pathogenic GRN mutations frequently show evidence of white-matter loss before symptom onset, ${ }^{97}$ and the risk allele of TMEM106B is associated with exacerbated disease-associated functional connectivity changes in presymptomatic $G R N$ carriers compared to healthy controls. ${ }^{21}$ These findings suggest that the modulation of FTLD risk by TMEM106B may be mediated not only via effects on circulating progranulin levels but also by modulating the severity of the whitematter phenotype observed in these individuals. Further, given what we know about the role of progranulin, these seemingly disparate effects may in fact be directly related to one another (Table 1).

A series of recently published papers have independently converged on the finding that loss of Tmem $106 \mathrm{~b}$ exacerbates a variety of neurodegeneration-associated phenotypes in mice also lacking Grn. ${ }^{9-101}$ Moreover, mice lacking both Tmem106b and Grn displayed exacerbated lysosomal dysfunction as well as signs of myelin damage. Indeed, loss of Tmem106b on a wild-type Grn background is sufficient to produce oligodendroglial and myelination defects, possibly downstream of lysosomal dysfunction. ${ }^{102,103}$ The whitematter abnormalities described in Tmem106b-deficient mice are not surprising given that pathogenic TMEM106B mutations have been identified as a cause of hypomyelinating leukodystrophy. ${ }^{104}$ In particular, a recurrent, dominant, and in some cases de novo mutation in TMEM106B has been found to cause a relatively mild form of hypomyelinating leukodystrophy. ${ }^{15,105}$

Given (i) the clear role of $G R N$ in maintaining lysosomal and microglial homeostasis; (ii) the established genetic interaction between GRN and TMEM106B; (iii) evidence of white-matter abnormalities in individuals with FTLD due to pathogenic $G R N$ mutations; and (iv) the importance of TMEM106B in lysosome function and myelination, it is apparent that the GRN-TMEM106B axis regulates white-matter integrity at least in part by promoting lysosomal and microglial homeostasis. In light of the above considerations, it is reasonable to speculate that heightened white-matter resilience in individuals harboring the protective allele of TMEM106B may represent a plausible mechanism for the modulation of FTLD risk due to pathogenic $G R N$ mutations. Interestingly, given that reductions in white-matter integrity have also been observed in C9orf72 pathogenic repeat expansion carriers, ${ }^{90,106}$ that $\mathrm{C} 9$ orf 72 protein also affects lysosomal function, ${ }^{107}$ and that $T M E M 106 B$ also modulates FTLD risk due to $C 9$ orf72 expansion, ${ }^{108,109}$ it is possible that white-matter resilience plays a role in the modulation of FTLD risk even beyond that contributed by GRN.

Early evidence from the Genetic FTD Initiative (GENFI) study suggests that, among individuals harboring pathogenic GRN mutations, those carrying the riskconferring variant in TMEM106B accrue white-matter changes more rapidly. ${ }^{20}$ Beyond these changes, greymatter volume analyses in autosomal dominant FTD (a combined cohort that included GRN, C9orf72, and MAPT mutation carriers from GENFI) found that TMEM106B genotype modulated the association between education and grey-matter volumes. ${ }^{110}$ Whether these grey-matter changes were preceded by or occurred in parallel with white-matter disturbances was not investigated but remains an exciting avenue for future research. Overall, these findings provide early evidence suggesting that multiple pathogenic hits to lysosomal and microglial homeostasis may confer susceptibility to and/or accelerate white-matter disease. ${ }^{20}$

\section{Prosaposin}

PSAP, encoding prosaposin, is genetically linked to several hereditary sphingolipidoses including metachromatic leukodystrophy, ${ }^{16}$ atypical forms of Krabbe disease ${ }^{111}$ and Gaucher disease, ${ }^{112}$ and combined prosaposin deficiency. ${ }^{113}$ Somewhat analogously to progranulin, prosaposin is synthesized as a precursor protein that, upon proteolysis, is converted into smaller proteins termed sphingolipid activator proteins or saposins. ${ }^{114}$ Pathogenic mutations in PSAP, generally found as homozygous or compound heterozygous variants, result in the loss of specific saposins and in some cases the entire precursor protein. As mentioned above, prosaposin is involved in the sorting of progranulin to the lysosome, ${ }^{115}$ and the loss of progranulin in turn impairs lysosomal delivery of prosaposin..$^{92}$ The impaired sorting and processing of prosaposin in $G R N$-mutant cells appears to result in reduced glucocerebrosidase activity, ${ }^{116,117}$ providing an interesting link to Gaucher disease and another potential mechanism that may contribute to disease risk in progranulinhaploinsufficient cells. Variation in the PSAP locus is 
also associated with circulating progranulin levels, which indicates an important genetic interaction in addition to the known biochemical interaction. ${ }^{118}$ Taken together, these functional connections between prosaposin and progranulin coupled with the clear genetic link between PSAP and leukodystrophy further reinforce the notion that progranulin-associated proteins are crucial for white-matter integrity (Table 1). Moreover, given what is known about $G R N$ and TMEM106B, it would not be surprising if variation in the PSAP locus is ultimately found to be associated with FTD risk as well.

\section{Sortilin}

In addition to prosaposin, the transmembrane protein sortilin is also involved in the delivery of progranulin to the lysosome. ${ }^{119}$ Quite interestingly, rare variation in SORT1, encoding sortilin, is now also implicated in risk for FTD. ${ }^{22}$ Moreover, a subset of the patients harboring rare, nonsynonymous variants in SORT1 show substantial WMH by neuroimaging, ${ }^{22}$ similar to what is frequently observed in FTLD due to pathogenic GRN mutations. Collectively, a consideration of the genetic and functional interactions between GRN, TMEM106B, PSAP, and SORT1; the known role of progranulin in promoting microglial homeostasis; and the association of this group of genes with various inherited white-matter disorders as well as forms of FTD often involving otherwise atypical white-matter findings suggests that these progranulin-associated genes shape microglial biology and bolster white-matter health during aging. By the same token, these genes illuminate underappreciated connections between white-matter resilience and risk for FTD (Table 1).

\section{Discussion and Future Directions}

In this review, we aimed to synthesize knowledge about and uncover connections between the primary microglial leukodystrophies - including Nasu-Hakola disease, ALSP, and pseudo-TORCH syndrome due to pathogenic USP 18 mutations - and inherited white-matter disorders such as adrenoleukodystrophy and Krabbe disease, in which microglia play an increasingly recognized role. In addition, we considered the literature surrounding progranulin and its functionally associated genes to draw connections between their roles in distinct leukodystrophies as well as forms of FTD involving otherwise atypical white-matter findings. The impact of progranulin on microglial and lysosomal physiology suggests that these cells and organelles are crucial for the facilitation of white-matter homeostasis. The loss of progranulin function, and that of progranulin-related proteins, highlights their role not only in traditionally recognized white-matter disorders but also in a seemingly unrelated disease - FTD - that nevertheless sometimes involves white-matter pathology in the absence of vascular risk factors.

Providing a rational basis for linking particular microglial phenotypes associated with pathogenic mutations to specific white-matter diseases and their typical ages of onset remains challenging. Nevertheless, we have summarized the current state of knowledge regarding the disorders discussed herein and the primary microglial phenotypes with which they are thought to be associated (Figure 1). Pseudo-TORCH syndrome due to USP18 mutations, representing a type I interferonopathy, leads to very early pathology, with signs of disease at or before birth. ${ }^{57}$ On the other hand, congenital absence of microglia observed in an individual harboring a homozygous splicesite mutation in CSFIR has also been associated with prenatal symptoms; an additional patient with a homozygous missense mutation in CSF1R showed symptom onset at age $12 .^{50}$ Histological studies of adrenoleukodystrophy, which has a wide age range of symptom onset, suggest that microglial activation, acquisition of an amoeboid phenotype, and loss of microglia may all be relevant cellular phenotypes. ${ }^{63-65}$ Moving to the adultonset, inherited white-matter disorders, evidence from mouse models suggests that Nasu-Hakola disease (due to loss of TREM2 or TYROBP) may be associated with heightened microglial susceptibility to apoptosis ${ }^{120}$ and impaired microglial lipid metabolism. ${ }^{40}$ Similarly, ALSP due to partial loss of $C S F 1 R$ may be associated with a reduction in microglia density, ${ }^{121}$ a shift toward an inflammatory microglial state, ${ }^{122}$ or both. Finally, FTLD due to $G R N$ haploinsufficiency, which often presents with white-matter pathology, may be associated with excessive complement production by microglia. ${ }^{12,14}$

Future studies of the primary microglial leukodystrophies should focus on determining precisely how alterations in seemingly disparate molecular pathways within microglia, such as those caused by pathogenic mutations in TREM2 and USP18, ultimately converge on the destruction of white matter. Further work in adrenoleukodystrophy and Krabbe disease will be needed to determine whether the therapeutic benefit observed for HSCT is derived from the engraftment of myeloid cells within an otherwise defective microglial niche, as is currently suspected. Evidence from mouse models suggests that under 


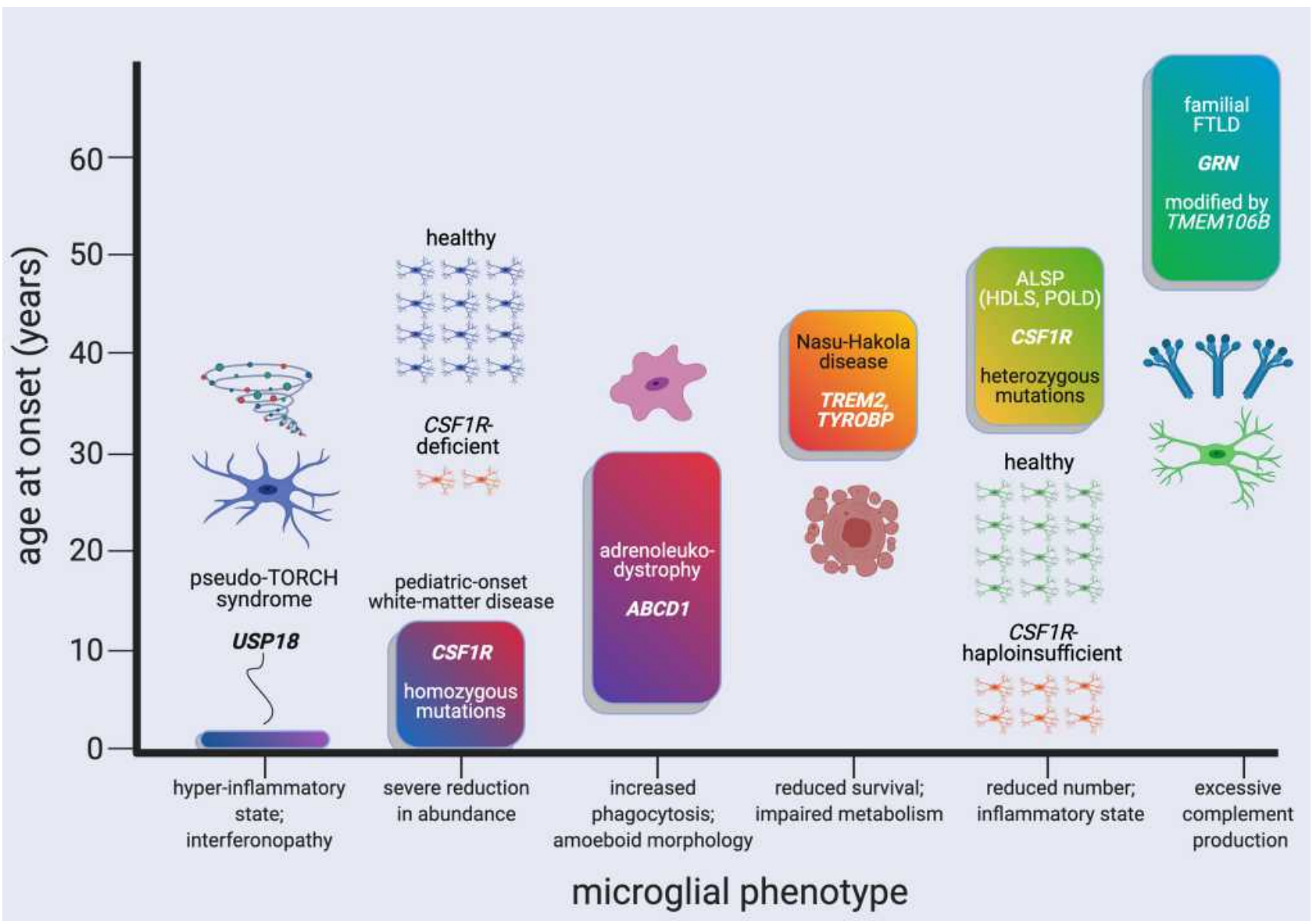

Figure I Distinct pathogenic mutations and microglial phenotypes are associated with white-matter disorders with highly variable ages of neurological symptom onset. White-matter diseases and the major microglial phenotypes they may be associated with are ordered according to their typical, approximate age range of onset. The characteristic microglial phenotypes listed are from histopathological studies and/or relevant mouse models of disease; see main text for references. Ages of neurological symptom onset can range from prenatal for type I interferonopathy associated with USPI8 deficiency and congenital absence (or near-absence) of microglia due to homozygous mutations in CSFIR, up to the 50 s-70s for some cases of frontotemporal lobar degeneration with white-matter hyperintensities associated with pathogenic GRN mutations. Created with BioRender.com.

Abbreviations: ABCDI, ATP-binding cassette DI; ALSP, adult-onset leukoencephalopathy with axonal spheroids and pigmented glia; CSFIR, colony-stimulating factor I receptor; FTLD, frontotemporal lobar degeneration; GRN, progranulin; HDLS, hereditary diffuse leukoencephalopathy with axonal spheroids; POLD, pigmented orthochromatic leukodystrophy; TMEMI06B, transmembrane protein 106B; TORCH, toxoplasmosis, other infections, rubella, cytomegalovirus, and herpes simplex virus; TREM2, triggering receptor expressed on myeloid cells 2; TYROBP, TYRO protein tyrosine kinase-binding protein; USPI8, ubiquitin-specific protease I8.

certain circumstances (such as acute ablation of microglia) peripheral myeloid cells are capable of efficiently migrating into the brain, where they acquire a similar - but not identical - phenotype to that of genuine microglia. ${ }^{123}$ Thus, it remains to be seen whether the benefits of HSCT in adrenoleukodystrophy and Krabbe disease are due to the restoration of $A B C D 1$ and $G A L C$ function, respectively, within brain-engrafted "microglia-like" cells derived from the transplant. Mouse models should enable this issue to be addressed in the future.

Finally, what are we to make of the WMH observed in a subset of patients with FTLD due to $G R N$ mutations or FTD associated with rare variation in SORT1? Should this subset of patients be considered to have an adultonset leukodystrophy? The extent of white-matter involvement in some cases seems to support this interpretation, although in these cases the white-matter pathology co-occurs with a prominent gray-matter structural phenotype and, presumably, TDP-43 neuropathology. Relatedly, it may be useful to consider the possibility that subtypes of FTLD-GRN exist, including those with and without extensive white-matter damage. Perhaps the involvement of white-matter pathology requires a "second hit," such as inheritance of the riskconferring allele of TMEM106B or concomitant reduction in the function of prosaposin or sortilin. Future discoveries in the genetics of FTD will determine whether there are additional, as-yet undiscovered connections between leukodystrophy and FTD, but the identification of variants in USP18 or PSAP, for example, that 
confer risk for FTD, would provide support for this intriguing possibility.

\section{Disclosure}

J.S.Y. reports this study was supported by NIH-NIA R01 AG062588, R01 AG057234, and the Rainwater Charitable Foundation. The authors report no other conflicts of interest in this work.

\section{References}

1. van der Knaap MS, Bugiani M. Leukodystrophies: a proposed classification system based on pathological changes and pathogenetic mechanisms. Acta Neuropathol (Berl). 2017;134 (3):351-382. doi:10.1007/s00401-017-1739-1

2. van der Knaap MS, Bugiani M. Leukodystrophies - much more than just diseases of myelin. Nat Rev Neurol. 2018;14 (12):747-748. doi:10.1038/s41582-018-0093-9

3. van der Knaap MS, Schiffmann R, Mochel F, Wolf NI. Diagnosis, prognosis, and treatment of leukodystrophies. Lancet Neurol. 2019;18(10):962-972. doi:10.1016/S1474-4422(19)30143-7

4. Schiller S, Henneke M, Gärtner J. Opening new horizons in the treatment of childhood onset leukodystrophies. Neuropediatrics. 2019;50(4):211-218. doi:10.1055/s-0039-1685529

5. Helman G, Venkateswaran S, Vanderver A. The spectrum of adult-onset heritable white-matter disorders. Handb Clin Neurol. 2018;148:669-692. doi:10.1016/B978-0-444-64076-5.00043-0

6. Lynch DS, Wade C, de Paiva ARB, et al. Practical approach to the diagnosis of adult-onset leukodystrophies: an updated guide in the genomic era. $J$ Neurol Neurosurg Psychiatry. 2019;90 (5):543-554. doi:10.1136/jnnp-2018-319481

7. Prinz M, Priller J. Microglia and brain macrophages in the molecular age: from origin to neuropsychiatric disease. Nat Rev Neurosci. 2014;15(5):300-312. doi:10.1038/nrn3722

8. Li Q, Barres BA. Microglia and macrophages in brain homeostasis and disease. Nat Rev Immunol. 2018;18(4):225-242. doi:10.1038/nri.2017.125

9. Song WM, Colonna M. The identity and function of microglia in neurodegeneration. Nat Immunol. 2018;19(10):1048-1058. doi:10.1038/s41590-018-0212-1

10. Kao AW, McKay A, Singh PP, Brunet A, Huang EJ. Progranulin, lysosomal regulation and neurodegenerative disease. Nat Rev Neurosci. 2017;18(6):325-333. doi:10.1038/nrn.2017.36

11. Paushter DH, Du H, Feng T, Hu F. The lysosomal function of progranulin, a guardian against neurodegeneration. Acta Neuropathol (Berl). 2018;136(1):1-17. doi:10.1007/s00401-0181861-8

12. Lui H, Zhang J, Makinson SR, et al. Progranulin deficiency promotes circuit-specific synaptic pruning by microglia via complement activation. Cell. 2016;165(4):921-935. doi:10.1016/j. cell.2016.04.001

13. Krabbe G, Minami SS, Etchegaray JI, et al. Microglial NFkB-TNF $\alpha$ hyperactivation induces obsessive-compulsive behavior in mouse models of progranulin-deficient frontotemporal dementia. Proc Natl Acad Sci U S A. 2017;114(19):5029-5034. doi:10.1073/pnas.1700477114

14. Zhang J, Velmeshev D, Hashimoto K, et al. Neurotoxic microglia promote TDP-43 proteinopathy in progranulin deficiency. Nature. 2020:1-5. doi:10.1038/s41586-020-2709-7.

15. Simons C, Dyment D, Bent SJ, et al. A recurrent de novo mutation in TMEM106B causes hypomyelinating leukodystrophy. Brain J Neurol. 2017;140(12):3105-3111. doi:10.1093/brain/ awx314
16. Cesani M, Lorioli L, Grossi S, et al. Mutation update of ARSA and PSAP genes causing metachromatic leukodystrophy. Hum Mutat. 2016;37(1):16-27. doi:10.1002/humu.22919

17. Cruts M, Gijselinck I, van der Zee J, et al. Null mutations in progranulin cause ubiquitin-positive frontotemporal dementia linked to chromosome 17q21. Nature. 2006;442(7105):920-924. doi:10.1038/nature05017

18. Baker M, Mackenzie IR, Pickering-Brown SM, et al. Mutations in progranulin cause tau-negative frontotemporal dementia linked to chromosome 17. Nature. 2006;442(7105):916-919. doi:10.1038/ nature 05016

19. Caroppo P, Le Ber I, Camuzat A, et al. Extensive white matter involvement in patients with frontotemporal lobar degeneration: think progranulin. JAMA Neurol. 2014;71(12):1562-1566. doi:10.1001/jamaneurol.2014.1316

20. Sudre $\mathrm{CH}$, Bocchetta M, Heller C, et al. White matter hyperintensities in progranulin-associated frontotemporal dementia: a longitudinal GENFI study. NeuroImage Clin. 2019;24:102077. doi:10.1016/j.nicl.2019.102077

21. Premi E, Formenti A, Gazzina S, et al. Effect of TMEM106B polymorphism on functional network connectivity in asymptomatic GRN mutation carriers. JAMA Neurol. 2014;71 (2):216-221. doi:10.1001/jamaneurol.2013.4835

22. Philtjens S, Van Mossevelde S, van der Zee J, et al. Rare nonsynonymous variants in SORT1 are associated with increased risk for frontotemporal dementia. Neurobiol Aging. 2018;66:181.e3181.e10. doi:10.1016/j.neurobiolaging.2018.02.011

23. Paloneva J, Kestilä M, Wu J, et al. Loss-of-function mutations in TYROBP (DAP12) result in a presenile dementia with bone cysts. Nat Genet. 2000;25(3):357-361. doi:10.1038/77153

24. Paloneva J, Manninen T, Christman G, et al. Mutations in two genes encoding different subunits of a receptor signaling complex result in an identical disease phenotype. Am J Hum Genet. 2002;71(3):656-662. doi:10.1086/342259

25. Ulland TK, Colonna M. TREM2 - a key player in microglial biology and Alzheimer disease. Nat Rev Neurol. 2018;12:1. doi:10.1038/s41582-018-0072-1

26. Paloneva J, Autti T, Raininko R, et al. CNS manifestations of Nasu-Hakola disease: a frontal dementia with bone cysts. Neurology. 2001;56(11):1552-1558. doi:10.1212/WNL.56.1 1.1552

27. Klünemann HH, Ridha BH, Magy L, et al. The genetic causes of basal ganglia calcification, dementia, and bone cysts: DAP12 and TREM2. Neurology. 2005;64(9):1502-1507. doi:10.1212/01. WNL.0000160304.00003.CA

28. Chouery E, Delague V, Bergougnoux A, Koussa S, Serre J-L, Mégarbané A. Mutations in TREM2 lead to pure early-onset dementia without bone cysts. Hum Mutat. 2008;29(9):E194E204. doi:10.1002/humu.20836

29. Guerreiro RJ, Lohmann E, Brás JM, et al. Using exome sequencing to reveal mutations in TREM2 presenting as a frontotemporal dementia-like syndrome without bone involvement. JAMA Neurol. 2013;70(1):78-84. doi:10.1001/ jamaneurol.2013.579

30. Guerreiro R, Bilgic B, Guven G, et al. Novel compound heterozygous mutation in TREM2 found in a Turkish frontotemporal dementia-like family. Neurobiol Aging. 2013;34(12):2890.e1-e5. doi:10.1016/j.neurobiolaging.2013.06.005

31. Borroni B, Ferrari F, Galimberti D, et al. Heterozygous TREM2 mutations in frontotemporal dementia. Neurobiol Aging. 2013. doi:10.1016/j.neurobiolaging.2013.09.017

32. Su W-H, Shi Z-H, Liu S-L, Wang X-D, Liu S, Ji Y. The rs75932628 and rs2234253 polymorphisms of the TREM2 gene were associated with susceptibility to frontotemporal lobar degeneration in Caucasian populations. Ann Hum Genet. 2018;82 (4):177-185. doi:10.1111/ahg.12241 
33. Jonsson T, Stefansson H, Steinberg S, et al. Variant of TREM2 associated with the risk of Alzheimer's disease. $N$ Engl J Med. 2013;368(2):107-116. doi:10.1056/NEJMoa1211103

34. Guerreiro R, Wojtas A, Bras J, et al. TREM2 variants in Alzheimer's disease. $N$ Engl $J$ Med. 2013;368(2):117-127. doi:10.1056/NEJMoa1211851

35. Colonna M, Wang Y. TREM2 variants: new keys to decipher Alzheimer disease pathogenesis. Nat Rev Neurosci. 2016;17 (4):201-207. doi:10.1038/nrn.2016.7

36. Ulrich JD, Ulland TK, Colonna M, Holtzman DM. Elucidating the Role of TREM2 in Alzheimer's Disease. Neuron. 2017;94 (2):237-248. doi:10.1016/j.neuron.2017.02.042

37. Yeh FL, Hansen DV, Sheng M. TREM2, microglia, and neurodegenerative diseases. Trends Mol Med. 2017;23(6):512-533. doi:10.1016/j.molmed.2017.03.008

38. Cantoni C, Bollman B, Licastro D, et al. TREM2 regulates microglial cell activation in response to demyelination in vivo. Acta Neuropathol (Berl). 2015;129(3):429-447. doi:10.1007/ s00401-015-1388-1

39. Poliani PL, Wang Y, Fontana E, et al. TREM2 sustains microglial expansion during aging and response to demyelination. J Clin Invest. 2015;125(5):2161-2170. doi:10.1172/JCI77983

40. Nugent AA, Lin K, van Lengerich B, et al. TREM2 regulates microglial cholesterol metabolism upon chronic phagocytic challenge. Neuron. 2019. doi:10.1016/j.neuron.2019.12.007

41. Cignarella F, Filipello F, Bollman B, et al. TREM2 activation on microglia promotes myelin debris clearance and remyelination in a model of multiple sclerosis. Acta Neuropathol (Berl). 2020;290 (686-698):26043. doi:10.1007/s00401-020-02193-z

42. Wider C, Van Gerpen JA, DeArmond S, Shuster EA, Dickson DW, Wszolek ZK. Leukoencephalopathy with spheroids (HDLS) and pigmentary leukodystrophy (POLD): a single entity? Neurology. 2009;72(22):1953-1959. doi:10.1212/WNL.0b013e 3181a826c0

43. Rademakers R, Baker M, Nicholson AM, et al. Mutations in the colony stimulating factor 1 receptor (CSF1R) gene cause hereditary diffuse leukoencephalopathy with spheroids. Nat Genet 2011;44(2):200-205. doi:10.1038/ng.1027

44. Nicholson AM, Baker MC, Finch NA, et al. CSF1R mutations link POLD and HDLS as a single disease entity. Neurology. 2013;80(11):1033-1040. doi:10.1212/WNL.0b013e31828726a7

45. Adams SJ, Kirk A, Auer RN. Adult-onset leukoencephalopathy with axonal spheroids and pigmented glia (ALSP): integrating the literature on hereditary diffuse leukoencephalopathy with spheroids (HDLS) and pigmentary orthochromatic leukodystrophy (POLD). J Clin Neurosci. 2018;48:42-49. doi:10.1016/j.jocn.2017.10.060

46. Ginhoux F, Greter M, Leboeuf M, et al. Fate mapping analysis reveals that adult microglia derive from primitive macrophages Science. 2010;330(6005):841-845. doi:10.1126/science.1194637

47. Wang Y, Szretter KJ, Vermi W, et al. IL-34 is a tissue-restricted ligand of CSF1R required for the development of Langerhans cells and microglia. Nat Immunol. 2012;13(8):753-760. doi: $10.1038 /$ ni.2360

48. Konno T, Kasanuki K, Ikeuchi T, Dickson DW, Wszolek ZK. CSF1R-related leukoencephalopathy: a major player in primary microgliopathies. Neurology. 2018;91(24):1092-1104 doi:10.1212/WNL.0000000000006642

49. Gelfand JM, Greenfield AL, Barkovich M, et al. Allogeneic HSCT for adult-onset leukoencephalopathy with spheroids and pigmented glia. Brain $J$ Neurol. 2020;143(2):503-511. doi:10.1093/brain/awz390

50. Oosterhof N, Chang IJ, Karimiani EG, et al. Homozygous mutations in CSF1R cause a pediatric-onset leukoencephalopathy and can result in congenital absence of microglia. Am J Hum Genet. 2019;104(5):936-947. doi:10.1016/j.ajhg.2019.03.010
51. Greter M, Lelios I, Pelczar P, et al. Stroma-derived interleukin-34 controls the development and maintenance of langerhans cells and the maintenance of microglia. Immunity. 2012;37(6):1050-1060. doi:10.1016/j.immuni.2012.11.001

52. Wu S, Xue R, Hassan S, et al. I134-Csf1r pathway regulates the migration and colonization of microglial precursors. Dev Cell. 2018;46(5):552-563.e4. doi:10.1016/j.devcel.2018.08.005

53. Dallabona C, Diodato D, Kevelam SH, et al. Novel (ovario) leukodystrophy related to AARS2 mutations. Neurology. 2014;82(23):2063-2071. doi:10.1212/WNL.0000000000000497

54. Lynch DS, Zhang WJ, Lakshmanan R, et al. Analysis of mutations in AARS2 in a series of CSF1R-negative patients with adult-onset leukoencephalopathy with axonal spheroids and pigmented glia. JAMA Neurol. 2016;73(12):1433-1439. doi:10.1001/ jamaneurol.2016.2229

55. Taglia I, Di Donato I, Bianchi S, et al. AARS2-related ovarioleukodystrophy: clinical and neuroimaging features of three new cases. Acta Neurol Scand. 2018;42(Supp13):S27. doi:10.1111/ane.12954

56. Goldmann T, Zeller N, Raasch J, et al. USP18 lack in microglia causes destructive interferonopathy of the mouse brain. EMBO J. 2015;34(12):1612-1629. doi:10.15252/embj.201490791

57. Meuwissen MEC, Schot R, Buta S, et al. Human USP18 deficiency underlies type 1 interferonopathy leading to severe pseudo-TORCH syndrome. J Exp Med. 2016;213(7):1163-1174. doi:10.1084/jem.20151529

58. Schwabenland M, Mossad O, Peres AG, et al. Loss of USP18 in microglia induces white matter pathology. Acta Neuropathol Commun. 2019;7(1):106. doi:10.1186/s40478-019-0757-8

59. Honke N, Shaabani N, Zhang D-E, Hardt C, Lang KS. Multiple functions of USP18. Cell Death Dis. 2016;7(11):e2444. doi:10.1038/cddis.2016.326

60. Takata K, Ginhoux F. Poised for action: USP18 restrains microglial activation in the white matter. EMBO J. 2015;34 (12):1603-1605. doi:10.15252/embj.201591899

61. Engelen M, Kemp S, Poll-The B-T. X-linked adrenoleukodystrophy: pathogenesis and treatment. Curr Neurol Neurosci Rep. 2014;14(10):486. doi:10.1007/s11910-014-0486-0

62. Huffnagel IC, Laheji FK, Aziz-Bose R, et al. The natural history of adrenal insufficiency in X-linked adrenoleukodystrophy: an International Collaboration. J Clin Endocrinol Metab. 2019;104 (1):118-126. doi:10.1210/jc.2018-01307

63. Gong Y, Sasidharan N, Laheji F, et al. Microglial dysfunction as a key pathological change in adrenomyeloneuropathy. Ann Neurol. 2017;82(5):813-827. doi:10.1002/ana.25085

64. Bergner CG, van der Meer F, Winkler A, et al. Microglia damage precedes major myelin breakdown in X-linked adrenoleukodystrophy and metachromatic leukodystrophy. Glia. 2019;67 (6):1196-1209. doi:10.1002/glia.23598

65. Eichler FS, Ren J-Q, Cossoy M, et al. Is microglial apoptosis an early pathogenic change in cerebral X-linked adrenoleukodystrophy? Ann Neurol. 2008;63(6):729-742. doi:10.1002/ana.21391

66. Shapiro E, Krivit W, Lockman L, et al. Long-term effect of bone-marrow transplantation for childhood-onset cerebral X-linked adrenoleukodystrophy. Lancet Lond Engl. 2000;356 (9231):713-718. doi:10.1016/S0140-6736(00)02629-5

67. Eichler F, Duncan C, Musolino PL, et al. Hematopoietic Stem-cell gene therapy for cerebral adrenoleukodystrophy. $N$ Engl J Med. 2017;377(17):1630-1638. doi:10.1056/NEJMoa1700554

68. Maegawa GHB. Lysosomal leukodystrophies lysosomal storage diseases associated with white matter abnormalities. J Child Neurol. 2019;34(6):339-358. doi:10.1177/0883073819828587

69. Suzuki K. Twenty five years of the "psychosine hypothesis": a personal perspective of its history and present status. Neurochem Res. 1998;23(3):251-259. doi:10.1023/ a: 1022436928925 
70. Graziano ACE, Cardile V. History, genetic, and recent advances on Krabbe disease. Gene. 2015;555(1):2-13. doi:10.1016/j. gene.2014.09.046

71. Nicaise AM, Bongarzone ER, Crocker SJ. A microglial hypothesis of globoid cell leukodystrophy pathology. J Neurosci Res. 2016;94(11):1049-1061. doi:10.1002/jnr.23773

72. Ijichi K, Brown GD, Moore CS, et al. MMP-3 mediates psychosine-induced globoid cell formation: implications for leukodystrophy pathology. Glia. 2013;61(5):765-777. doi:10.1002/ glia.22471

73. Potter GB, Santos M, Davisson MT, et al. Missense mutation in mouse GALC mimics human gene defect and offers new insights into Krabbe disease. Hum Mol Genet. 2013;22(17):3397-3414. doi: $10.1093 / \mathrm{hmg} / \mathrm{ddt} 190$

74. Giri S, Khan M, Rattan R, Singh I, Singh AK. Krabbe disease: psychosine-mediated activation of phospholipase A2 in oligodendrocyte cell death. J Lipid Res. 2006;47(7):1478-1492. doi:10.1194/jlr.M600084-JLR200

75. Ida H, Rennert OM, Watabe K, Eto Y, Maekawa K. Pathological and biochemical studies of fetal Krabbe disease. Brain Dev. 1994;16(6):480-484. doi:10.1016/0387-7604(94)90013-2

76. Krivit W, Shapiro EG, Peters C, et al. Hematopoietic stem-cell transplantation in globoid-cell leukodystrophy. $N$ Engl $\mathrm{J}$ Med. 1998;338(16):1119-1126. doi:10.1056/NEJM199804163381605

77. Escolar ML, Poe MD, Provenzale JM, et al. Transplantation of umbilical-cord blood in babies with infantile Krabbe's disease. $N$ Engl J Med. 2005;352(20):2069-2081. doi:10.1056/ NEJMoa042604

78. McGraw P, Liang L, Escolar M, Mukundan S, Kurtzberg J, Provenzale JM. Krabbe disease treated with hematopoietic stem cell transplantation: serial assessment of anisotropy measurements-initial experience. Radiology. 2005;236(1):221-230. doi:10.1148/radiol.2353040716

79. Giera S, Luo R, Ying Y, et al. Microglial transglutaminase-2 drives myelination and myelin repair via GPR56/ADGRG1 in oligodendrocyte precursor cells. eLife. 2018;7. doi:10.7554/ eLife. 33385

80. Wang J, He X, Meng H, et al. Robust myelination of regenerated axons induced by combined manipulations of GPR17 and microglia. Neuron. 2020;108(5):876-886.e4. doi:10.1016/j. neuron.2020.09.016

81. Lund H, Pieber M, Parsa R, et al. Fatal demyelinating disease is induced by monocyte-derived macrophages in the absence of TGF- $\beta$ signaling. Nat Immunol. 2018;19(5):1-7. doi:10.1038/ s41590-018-0091-5

82. Qin Y, Garrison BS, Ma W, et al. A milieu molecule for TGF- $\beta$ required for microglia function in the nervous system. Cell. 2018;174(1):156-171.e16. doi:10.1016/j.cell.2018.05.027

83. Arnold TD, Lizama CO, Cautivo KM, et al. Impaired $\alpha \mathrm{V} \beta 8$ and TGF $\beta$ signaling lead to microglial dysmaturation and neuromotor dysfunction. J Exp Med. 2019;216(4):900-915. doi:10.1084/ jem. 20181290

84. Li Q, Cheng Z, Zhou L, et al. Developmental heterogeneity of microglia and brain myeloid cells revealed by deep single-cell RNA sequencing. Neuron. 2019;101(2):207-223.e10. doi:10.1016/j.neuron.2018.12.006

85. Safaiyan S, Besson-Girard S, Kaya T, et al. White matter aging drives microglial diversity. Neuron. 2021. doi:10.1016/j. neuron.2021.01.027

86. Sirkis DW, Geier EG, Bonham LW, Karch CM, Yokoyama JS. Recent advances in the genetics of frontotemporal dementia. Curr Genet Med Rep. 2019;7(1):41-52. doi:10.1007/s40142-019-0160-6

87. Mahoney CJ, Simpson IJA, Nicholas JM, et al. Longitudinal diffusion tensor imaging in frontotemporal dementia. Ann Neurol. 2015;77(1):33-46. doi:10.1002/ana.24296
88. Jiskoot LC, Bocchetta M, Nicholas JM, et al. Presymptomatic white matter integrity loss in familial frontotemporal dementia in the GENFI cohort: a cross-sectional diffusion tensor imaging study. Ann Clin Transl Neurol. 2018;5(9):1025-1036. doi:10.1002/acn3.601

89. Le Ber I, Camuzat A, Hannequin D, et al. Phenotype variability in progranulin mutation carriers: a clinical, neuropsychological, imaging and genetic study. Brain $J$ Neurol. 2008;131(Pt 3):732-746. doi:10.1093/brain/awn012

90. Ameur F, Colliot O, Caroppo P, et al. White matter lesions in FTLD: distinct phenotypes characterize GRN and C9ORF72 mutations. Neurol Genet. 2016;2(1):e47. doi:10.1212/ NXG.0000000000000047

91. Woollacott IOC, Bocchetta M, Sudre CH, et al. Pathological correlates of white matter hyperintensities in a case of progranulin mutation associated frontotemporal dementia. Neurocase. 2018;24(3):166-174. doi:10.1080/13554794.2018.1506039

92. Zhou X, Sun L, Bracko O, et al. Impaired prosaposin lysosomal trafficking in frontotemporal lobar degeneration due to progranulin mutations. Nat Commun. 2017;8(1):1-14. doi:10.1038/ ncomms 15277

93. Götzl JK, Brendel M, Werner G, et al. Opposite microglial activation stages upon loss of PGRN or TREM2 result in reduced cerebral glucose metabolism. EMBO Mol Med. 2019;11(6). doi:10.15252/emmm.201809711

94. Cruchaga C, Graff C, Chiang - $\mathrm{H}-\mathrm{H}$, et al. Association of TMEM106B gene polymorphism with age at onset in granulin mutation carriers and plasma granulin protein levels. Arch Neurol. 2011;68(5):581-586. doi:10.1001/archneurol.2010.350

95. Finch N, Carrasquillo MM, Baker M, et al. TMEM106B regulates progranulin levels and the penetrance of FTLD in GRN mutation carriers. Neurology. 2011;76(5):467-474. doi:10.1212/ WNL.0b013e31820a0e3b

96. van der Zee J, Van Langenhove $\mathrm{T}$, Kleinberger $\mathrm{G}$, et al. TMEM106B is associated with frontotemporal lobar degeneration in a clinically diagnosed patient cohort. Brain J Neurol. 2011;134 (Pt3):808-815. doi:10.1093/brain/awr007

97. Dopper EGP, Rombouts SARB, Jiskoot LC, et al. Structural and functional brain connectivity in presymptomatic familial frontotemporal dementia. Neurology. 2014;83(2):e19-e26. doi:10.1212/ WNL.0000000000000583

98. Feng T, Mai S, Roscoe JM, et al. Loss of TMEM106B and PGRN leads to severe lysosomal abnormalities and neurodegeneration in mice. EMBO Rep. 2020;127(10):e50219. doi:10.15252/ embr.202050219

99. Werner G, Damme M, Schludi M, et al. Loss of TMEM106B potentiates lysosomal and FTLD-like pathology in progranulin-deficient mice. EMBO Rep. 2020;21(10):e50241. doi:10.15252/embr.202050241

100. Zhou X, Brooks M, Jiang P, et al. Loss of Tmem106b exacerbates FTLD pathologies and causes motor deficits in progranulin-deficient mice. EMBO Rep. 2020;39:e50197. doi:10.15252/embr.202050197

101. Clayton EL, Isaacs AM. Progranulin and TMEM106B: when two become wan. EMBO Rep. 2020;21(10):e51668. doi:10.15252/ embr.202051668

102. Zhou X, Nicholson AM, Ren Y, et al. Loss of TMEM106B leads to myelination deficits: implications for frontotemporal dementia treatment strategies. Brain J Neurol. 2020;61(6):435-1919. doi:10.1093/brain/awaa141

103. Feng $T$, Sheng RR, Solé-Domènech $S$, et al. A role of the frontotemporal lobar degeneration risk factor TMEM106B in myelination. Brain J Neurol. 2020;143(7):2255-2271. doi:10.1093/brain/awaa154 
104. Zhou X, Rademakers R. TMEM106B and myelination: rare leukodystrophy families reveal unexpected connections. Brain $J$ Neurol. 2017;140(12):3069-3080. doi:10.1093/brain/awx318

105. Yan H, Kubisiak T, Ji H, Xiao J, Wang J, Burmeister M. The recurrent mutation in TMEM106B also causes hypomyelinating leukodystrophy in China and is a CpG hotspot. Brain J Neurol. 2018;141(5):e36. doi:10.1093/brain/awy029

106. Panman JL, Jiskoot LC, Bouts MJRJ, et al. Gray and white matter changes in presymptomatic genetic frontotemporal dementia: a longitudinal MRI study. Neurobiol Aging. 2019;76:115-124. doi:10.1016/j.neurobiolaging.2018.12.017

107. Amick J, Ferguson SM. C9orf72: at the intersection of lysosome cell biology and neurodegenerative disease. Traffic Cph Den. 2017;18(5):267-276. doi:10.1111/tra.12477

108. van Blitterswijk M, Mullen B, Nicholson AM, et al. TMEM106B protects C9ORF72 expansion carriers against frontotemporal dementia. Acta Neuropathol (Berl). 2014;127(3):397-406. doi:10.1007/s00401-013-1240-4

109. Gallagher MD, Suh E, Grossman M, et al. TMEM106B is a genetic modifier of frontotemporal lobar degeneration with C9orf72 hexanucleotide repeat expansions. Acta Neuropathol (Berl). 2014;127(3):407-418. doi:10.1007/s00401-013-1239-x

110. Premi E, Grassi M, van Swieten J, et al. Cognitive reserve and TMEM106B genotype modulate brain damage in presymptomatic frontotemporal dementia: a GENFI study. Brain $J$ Neurol. 2017;140(6):1784-1791. doi:10.1093/brain/awx103

111. Spiegel R, Bach G, Sury V, et al. A mutation in the saposin A coding region of the prosaposin gene in an infant presenting as Krabbe disease: first report of saposin A deficiency in humans. Mol Genet Metab. 2005;84(2):160-166. doi:10.1016/j.ymgme.20 04.10 .004

112. Vaccaro AM, Motta M, Tatti M, et al. Saposin C mutations in Gaucher disease patients resulting in lysosomal lipid accumulation, saposin $\mathrm{C}$ deficiency, but normal prosaposin processing and sorting. Hum Mol Genet. 2010;19(15):2987-2997. doi:10.1093/ $\mathrm{hmg} / \mathrm{ddq} 204$

113. Motta M, Tatti M, Furlan F, et al. Clinical, biochemical and molecular characterization of prosaposin deficiency. Clin Genet. 2016;90(3):220-229. doi:10.1111/cge.12753
114. Kolter T, Sandhoff K. Principles of lysosomal membrane digestion: stimulation of sphingolipid degradation by sphingolipid activator proteins and anionic lysosomal lipids. Annu Rev Cell Dev Biol. 2005;21(1):81-103. doi:10.1146/annurev. cellbio.21.122303.120013

115. Zhou X, Sun L, Bastos de Oliveira F, et al. Prosaposin facilitates sortilin-independent lysosomal trafficking of progranulin. J Cell Biol. 2015;210(6):991-1002. doi:10.1083/jcb.201502029

116. Arrant AE, Roth JR, Boyle NR, et al. Impaired $\beta$ glucocerebrosidase activity and processing in frontotemporal dementia due to progranulin mutations. Acta Neuropathol Commun. 2019;7(1):1-17. doi:10.1186/s40478-019-0872-6

117. Valdez C, Ysselstein D, Young TJ, Zheng J, Krainc D. Progranulin mutations result in impaired processing of prosaposin and reduced glucocerebrosidase activity. Hum Mol Genet. 2020;29(5):716-726. doi:10.1093/hmg/ddz229

118. Nicholson AM, Finch NA, Almeida M, et al. Prosaposin is a regulator of progranulin levels and oligomerization. Nat Commun. 2016;7(1):11992. doi:10.1038/ncomms11992

119. Hu F, Padukkavidana T, Vægter CB, et al. Sortilin-mediated endocytosis determines levels of the frontotemporal dementia protein, progranulin. Neuron. 2010;68(4):654-667. doi:10.1016/ j.neuron.2010.09.034

120. Wang Y, Cella M, Mallinson K, et al. TREM2 lipid sensing sustains the microglial response in an Alzheimer's Disease Model. Cell. 2015;160(6):1061-1071. doi:10.1016/j. cell.2015.01.049

121. Oosterhof N, Kuil LE, van der Linde HC, et al. Colonystimulating factor 1 receptor (CSF1R) regulates microglia density and distribution, but not microglia differentiation in vivo. Cell Rep. 2018;24(5):1203-1217.e6. doi:10.1016/j.celrep.2018.06.113

122. Kempthorne L, Yoon H, Madore C, et al. Loss of homeostatic microglial phenotype in CSF1R-related Leukoencephalopathy. Acta Neuropathol Commun. 2020;8(1):1-15. doi:10.1186/ s40478-020-00947-0

123. Prokop S, Miller KR, Drost N, et al. Impact of peripheral myeloid cells on amyloid- $\beta$ pathology in Alzheimer's disease-like mice. J Exp Med. 2015;212(11):1811-1818. doi:10.1084/jem.20150479
The Application of Clinical Genetics

\section{Publish your work in this journal}

The Application of Clinical Genetics is an international, peerreviewed open access journal that welcomes laboratory and clinical findings in the field of human genetics. Specific topics include: Population genetics; Functional genetics; Natural history of genetic disease; Management of genetic disease; Mechanisms of genetic disease;

\section{Dovepress}

Counselling and ethical issues; Animal models; Pharmacogenetics; Prenatal diagnosis; Dysmorphology. The manuscript management system is completely online and includes a very quick and fair peerreview system, which is all easy to use. Visit http://www.dovepress. com/testimonials.php to read real quotes from published authors. 